\title{
ALGUNS HELMINTOS DE PEIXES DO LITORAL NORTE FLUMINENSE - II. ${ }^{1}$
}

\author{
J. JULIO VICENTE* e ELIAS DOS SANTOS**
}

(Com 11 figuras)

SUMĀRIO: De 62 autópsias realizadas em peixes, capturados no litoral de Macaé, Estado do Rio de Janeiro, os autores coletaram alguns exemplares de helmintos cujo estudo permitiu a descrição de 2 espécies novas: Cucullanus rougetae e Raphidascaris yamagutii; a redescrição de Pseudopecoelus elongatus (Yamaguti, 1938) em novo hospedeiro, as referências de Procamallanus macaensis Vicente e Santos, 1972, e Catarinatrema verrucosum Freitas \& Santos, 1971 e a descrição de 2 larvas de nematódeos e 1 de cestódeo que não puderam ser determinadas até espécie.

A excursão em que foram colecionados os helmintos acima relacionados foi realizada em janeiro de 1973 por um de nós (Elias dos Santos) permitindo a continuação de trabalho anterior sobre helmintos de peixes marinhos daquele município fluminense.

\section{MATERIAL E MÉTODOS}

Os helmintos coletados após as necrópsias, foram recolhidos em soro fisiológico e depois fixados em líquido de Railliet \& Henry. Os nematódeos foram fixados a quente, diafanizados em creosoto de faia e montados em bálsamo do Canadá; os trematódeos e larvas de cestódeos, foram fixados a frio, comprimidos entre lâmina e lamínula, corados pelo carmim clorídrico-alcoólico, diafanizados em creosoto de faia e montados em bálsamo do Canadá.

\section{RESULTADOS}

NEMATODA

Camallanidae Railliet \& Henry, 1915
Gênero Procamallanus Baylis, 1923

Procamallanus macaensis Vicente \& Santos, 1972

Referências - Procamallanus macaensis: Vicente \& Santos, 1972: 145-147, 5 figs.; Procamallanus maca. ensis: Vicente \& Santos, 1973: 95.

Habitat - Cecos de Urophycis sp. Brasil.

Proveniência - Macaé, Estado do Rio de Janeiro,

Material estudado, depositado na Coleção Helmintológica do Instituto Oswaldo Cruz, sob o número 30968 a-d.

Referências bibliográficas - 22, 23.

Observação - Referido em novo hospedeiro.

Cucullanidae Cobbold, 1864

Gênero Cucullanus Mueller, 1777

1 Entregue para publicação em 27 de dezembro de 1973.

* Pesquisador do Instituto Oswaldo Cruz e Bolsista do CNPq.

** Bolsista do CNPq. Laboratório de Helmintologia do Departamento de Zoologia Médica. 
QUADRO I

\begin{tabular}{|c|c|c|c|c|c|c|c|}
\hline NOME VULGAR & NOME CIENTÍFICO & $\begin{array}{c}\text { N.O DE } \\
\text { NECROPSIAS }\end{array}$ & $\begin{array}{l}\text { NECROPSIAS } \\
\text { POSITIVAS }\end{array}$ & $\begin{array}{l}\text { NECROPSIAS } \\
\text { NEGATIVAS }\end{array}$ & FREQUUÊNCIA & ESPECIE & $\begin{array}{c}\text { N. }{ }^{\circ} \text { DE } \\
\text { EXEMPLARES }\end{array}$ \\
\hline \multirow[t]{2}{*}{ Peixe espada } & \multirow[t]{2}{*}{ Trichiurus lepturus $L$. } & \multirow[t]{2}{*}{5} & \multirow[t]{2}{*}{3} & \multirow[t]{2}{*}{2} & \multirow[t]{2}{*}{$60 \%$} & Catarinatrema verrucosum & 6 \\
\hline & & & & & & Pseudopecoelus elongatus & 10 \\
\hline Bagre calafate & Tachysurus sp. & 5 & - & 5 & - & - & - \\
\hline Cabeça dura & - & 1 & - & 1 & - & - & - \\
\hline Sardinha boca rasgada & Anchovia sp. & 4 & - & 4 & - & - & - \\
\hline Cavalinha do reino & Scomber colias & 3 & 1 & 2 & $33,3 \%$ & Raphidascaris sp. 1 & 10 \\
\hline Sardinha maromba & - & 6 & 1 & 5 & $16,6 \%$ & Raphidascaris sp. 2 & 1 \\
\hline Enxada & Chaetodipterus faber & 5 & - & 5 & - & - & - \\
\hline Solteira & Scombroides occidentalis & 3 & - & 3 & - & - & - \\
\hline Bonito serra & Euthynnus alletteratus & 2 & - & 2 & - & - & - \\
\hline Cação & Squatina squatina & 3 & - & 3 & - & - & - \\
\hline Bonito chachorro & - & 1 & - & 1 & - & - & - \\
\hline Cação martelo & - & 4 & - & 4 & - & - & - \\
\hline Peixe voador & Cypsilurus sp. & 1 & - & 1 & - & - & - \\
\hline Bagre guri & - & 2 & - & 2 & - & - & - \\
\hline Cabrinha & Prionotus sp. & 1 & 1 & - & $100 \%$ & Phyllobotridae sp. & 2 \\
\hline Enchova & Chaedipterus salttator. & 1 & - & 1 & - & - & - \\
\hline Pescadinha cabuçu & Plagioscion squamossimus & 1 & - & 1 & - & - & - \\
\hline Pescadinha rolão & Plagioscion auratus & 2 & 1 & 1 & $50 \%$ & Cucullanus rougetae sp. $\mathrm{n}$. & 1 \\
\hline \multirow[t]{2}{*}{ Abrotea } & \multirow[t]{2}{*}{ Urophycis sp. } & \multirow[t]{2}{*}{2} & \multirow[t]{2}{*}{2} & \multirow[t]{2}{*}{-} & \multirow[t]{2}{*}{$100 \%$} & Procamallanus macaensis & 4 \\
\hline & & & & & & Phyllobotridae sp. & 3 \\
\hline Guéte & - & 3 & - & 3 & - & - & - \\
\hline Faneca & - & 3 & - & 3 & - & - & - \\
\hline Bagre bandeira & Felichthys sp. & 1 & - & 1 & - & - & - \\
\hline Papa-terra & Menticirrus americanus & 1 & - & 1 & - & - & - \\
\hline Pescadinha & Nebris microps & 1 & 1 & - & $100 \%$ & Raphidascaris yamagutii sp. n. & 4 \\
\hline Bacalhau & - & 1 & 1 & - & $100 \%$ & - & - \\
\hline
\end{tabular}


Cucullanus rougetae sp. $\mathrm{n}$. (Figs. 1-4)

Comprimento - Macho 3,71 mm.

Largura - Macho 0,30 $\mathrm{mm}$.

Corpo cilíndrico de coloração branco-amarelada em vida, com cutícula estriada transversalmente. Extremidade anterior truncada e posterior cônica. Papilas cefálicas em número de três pares: 1 lateral, 1 látero-dorsal e 1 látero-ventral. Boca bilabiada, cada lábio possuindo em seu bordo interno uma fileira de dentículos bem nítidos. Esôfago musculoso em forma de maçã, mais alargado nas extremidades, medindo $0,63 \mathrm{~mm}$ de comprimento. $\hat{\mathrm{E}}$ separado do intestino por válvulas em forma de campânula. Intestino retilíneo. Anel nervoso situado a $0,21 \mathrm{~mm}$ da extremidade anterior.

Espículos iguais e semelhantes, medindo $0,96 \mathrm{~mm}$ de comprimento. Gubernáculo pequeno, pouco quitinizado com $0,066 \mathrm{~mm}$ de comprimento. Asas caudais ausentes. Papilas caudais em nú mero de 11 pares assim distribuídos: 6 pré-anais e 5 pós-anais. Extremidade posterior um pouco curvada ventralmente. Ânus situado a $0,10 \mathrm{~mm}$ do ápice caudal. Tubo genital dirigido para diante. Cloaca curta. Canal ejaculador forte. Testículo alcançando as proximidades do fim do esôfago. $O$ ápice caudal apresenta uma pequena formação em forma de espinho com 3 pontas e mede $0,014 \mathrm{~mm}$ de comprimento.

\section{Habitat - Intestino de Plagioscion auratus.}

Proveniência - Macaé, Estado do Rio de Janeiro, Brasil.

Holótipo número 30969 depositado na Coleção Helmintológica do Instituto Oswaldo Cruz.

O nome específico é dado em homenagem à Helmintologista francesa IVONNE CAMPANA-ROUGET.

DISCUSSĀO - Cucullanus rougetae sp. n. diferencia-se nitidamente das demais espécies do gênero por não apresentar pseudoventosa pré-anal e pela présença de uma formação quitinizada terminal em forma de espinho de três pontas.

Referências bibliográficas $-1,2,3,6,8,11,13$, 16,19 e 20.

Heterocheilidae Railliet \& Henry, 1915

Gênero Raphidascaris Railliet \& Henry, 1915

Raphidascaris yamagutii sp. $\mathrm{n}$. (Figs. 5-7)

Comprimento - Machos 3,60 a 3,91 mm; fêmeas 4,59 a $6,49 \mathrm{~mm}$.

Largura - Machos 0,23 a 0,30 mm; fêmeas 0,22 a $0,34 \mathrm{~mm}$.
Nematódeos alongados, afilados nas extremidades com cutícula finamente estriada transversalmente. Boca trilabiada, cada lábio com 1 par de papilas. Esôfago com 0,63 a $0,64 \mathrm{~mm}$ de comprimento nos machos e 0,63 a $0,67 \mathrm{~mm}$ nas fêmeas. Ventrículo medindo 0,042 a $0,054 \mathrm{~mm}$ de comprimento por 0,09 a $0,10 \mathrm{~mm}$ de largura nos machos e 0,042 a $0,063 \mathrm{~mm}$ de comprimento por 0,091 a $0,13 \mathrm{~mm}$ de largura nas fêmeas. Divertículo ventricular dirigido para trás medindo $0,20 \mathrm{~mm}$ de comprimento nos machos e 0,27 a $0,32 \mathrm{~mm}$ nas fêmeas. Anel nervoso distundo 0,16 a $0,17 \mathrm{~mm}$ da extremidade anterior nos machos e 0,17 a $0,19 \mathrm{~mm}$ nas fêmeas. Poro excretor a $0,16 \mathrm{~mm}$ do ápice cefálico nos machos e $0,21 \mathrm{~mm}$ nas fêmeas.

Fêmeas didelfas, anfidelfas, com vulva em forma de fenda transversal distando 1,15 a $1,61 \mathrm{~mm}$ da extremidade anterior. Ovejetor longo, musculoso, dirigido para trás e medindo $1,19 \mathrm{~mm}$ de comprimento. Ovos arredondados medindo $0,014 \mathrm{~mm}$ de comprimento por $0,013 \mathrm{~mm}$ de largura. Ânus situado 0,12 a $0,26 \mathrm{~mm}$ da extremidade posterior. Reto medindo 0,090 a $0,10 \mathrm{~mm}$ de comprimento. $O$ ápice caudal apresenta-se repleto de pequenos espinhos. Estreita asa caudal presente.

Machos com extremidade caudal curvada ventralmente. Papilas caudais pequenas, numerosas em número de aproximadamente 30 pares assim distribuídos: 20 pré-anais e 10 pós-anais. Espículos pequenos, iguais medindo $0,14 \mathrm{~mm}$ de comprimento. Ânus distando 0,07 a $0,08 \mathrm{~mm}$ do ápice caudal.

Habitat - Intestino de Nebris microps.

Proveniência - Macaé, Estado do Rio de Janeiro, Brasil.

Material estudado, depositado na Coleção Helmintológica do Instituto Oswaldo Cruz, sob o mímero 30970 a-d. Holótipo macho n.0 30970 d; holótipo fêmea n.0 $30970 \mathrm{~b}$.

$O$ nome específico é dado em homenagem ao grande helmintologista japonês SATYU YAMAGUTI.

Referências bibliográficas - 4, 7, 12, 14, 15 e 25 .

DISCUSSĀO - Estão incluídas atualmente nớ gênero Raphidascaris Railliet \& Henry, 1915, as seguintes espécies: $R$. acus (Bloch, 1779) Railliet \& Henry, 1915; $R$. alius Lyster, 1940; $R$. anchoviellae Chandler, 1935; $R$ biwakoensis Fujita, 1928; $R$. canadensis Smedley, 1933; $R$. cayugensis (Wigdor, 1918) York \& Maplestome, 1926; $\boldsymbol{R}$. chirocentri Yamaguti, 1935; $\boldsymbol{R}$. cristata (Linstow, 1872); $R$ gigi Fujita, 1928; $R$ gracillima (Linstow, 1890) Skrjabin, 1923; $R$. laurentianus Richardson, 1937; $R$ lutiani Olsen, 1952; $R$. panijii Khan \& Yassen, 1968; $R$. plecoglossi Fujita, 1928 e $R$ vicentei Santos, 1970.

O gênero Raphidascaris é mal organizado e muitas de suas descrições específicas são insuficientes e inadequadas. Várias espécies são conhecidas apenas por fêmeas, muitas vezes imaturas. Torna-se, portanto, extremamente difícil a determinação específica de exemplares deste grupo de nematódeos. 
Embora não tenhamos obtido algumas das descrições originais, orien tamos nossos estudos pelo trabalho básico de MOZGOVOI (1953) sobre os Ascaridoidea.

Das espécies descritas para o gênero Raphidascaris Railliet \& Henry, 1915, R. yamagutti sp. n. mais se aproxima de $R$. chirocentri Yamaguti, 1935, dela diferindo pelas dimensões de machos e fêmeas, pelo tamanho dos espículos, pelo número de papilas caudais do macho e pelas dimensões dos ovos, além de outros caracteres de menor interesse.

\section{Raphidascaris sp. 1}

(Fig. 8)

Comprimento - Fêmeas 6,18 a $6,49 \mathrm{~mm}$

Largu ra - Fêmeas 0,22 a $0,25 \mathrm{~mm}$

Nematódeos alongados, afilados nas extremidades com cutícula finamente estriada transversalmente. Boca trilabiada, cada lábio com 1 par de papilas. Esôfago com 0,70 a $0,71 \mathrm{~mm}$ de comprimento. Ventrículo medindo 0,025 a $0,030 \mathrm{~mm}$ de comprimento por 0,068 a $0,090 \mathrm{~mm}$ de largura. Ceco ventricular presente, dirigido para trás, medindo 0,38 a $0,40 \mathrm{~mm}$ de comprimento. Anel nervoso distando 0,25 a $0,28 \mathrm{~mm}$ da extremidade cefálica. Vulva situada no terço anterior do corpo, distando $1,60 \mathrm{~mm}$ da extremidade anterior. Ovejetor medindo $0,66 \mathrm{~mm}$ de comprimento. Reto com 0,090 a $0,10 \mathrm{~mm}$ de comprimento. Ânus distando 0,12 a $0,23 \mathrm{~mm}$ da extremidade posterior.

Habitat - Intestino de Scomber colias.

Proveniência - Macaé, Estado do Rio de Janeiro, Brasil.

Material estudado, depositado na Coleção Helmintológica do Instituto Oswaldo Cruz, sob o número $30971 \mathrm{a}-\mathrm{j}$.

Observações - Estudo realizado com fêmeas jovens (larvas de $4.0^{\circ}$ estádio).

Referência bibliográfica - 12 .

\section{Raphidascaris sp. 2}

(Fig. 9)

Comprimento - Fêmea 15,91 mm

Largura - Fêmea $0,68 \mathrm{~mm}$

Nematódeo alongado, afilado nas extremidades, com cutícula finamente estriada transversalmente. Boca trilabiada, cada lábio com 1 par de papilas e medindo $0,09 \mathrm{~mm}$ de comprimento. Esôfago com $1,66 \mathrm{~mm}$ de comprimento. Ventrículo com $0,14 \mathrm{~mm}$ de comprimento por $0,22 \mathrm{~mm}$ de largura. Ceco ventricular, dirigido para trás, medindo $0,63 \mathrm{~mm}$ de comprimento. Anel nervoso distando $0,42 \mathrm{~mm}$ da extremidade cefálica. Poro excretor situado um pouco para trás da região do anel nervoso, distando $0,63 \mathrm{~mm}$ da extremidade anterior. Asa lateral presente. Vulva situada no terço anterior do corpo distando $3,22 \mathrm{~mm}$ da extremidade anterior. Ovejetor medindo $1,82 \mathrm{~mm}$ de comprimento. Reto com $0,26 \mathrm{~mm}$ de comprimento. Ovos mais ou menos arredondados, medindo $0,039 \mathrm{~mm}$ de comprimento por $0,032 \mathrm{~mm}$ de largura. Ânus distando $0,35 \mathrm{~mm}$ do ápice caudal.

Habitat - Intestino de sardinha maromba.

Proveniência - Macaé, Estado do Rio de Janeiro, Brasil.

Referência bibliográfica -12 .

Observação - Estudo realizado com 1 fêmea jovem, que se extraviou após o estudo.

\section{TREMATODA}

Lecithochiriidae Skrjabin \& Guschanskaja, 1954

Gênero Catarinatrema Freitas \& Santos, 1971

Catarinatrema verrucosum Freitas \& Santos, 1971

Referência - Catarinatrema verrucosum: Freitas \& Santos, 1971: 79-81, 5 ests.

Habitat - Intestinos e cecos de Trichiurus lepturus $\mathrm{L}$.

Proveniência - Macaé, Estado do Rio de Janeiro, Brasil.

Material estudado, depositado na Coleção Helmintológica do Instituto Oswaldo Cruz, sob o número 30973 a-f.

Referência bibliográfica -5 .

Opecoelidae Ozaki, 1925

Gênero Pseudopecoelus Wicklen, 1946

Pseudopecoelus elongatus (Yamaguti, 1938)

Wicklen, 1946

(Fig. 10)

Referências e sinonímia - Cymbephallus elongatus Yamaguti, 1938: 50-51, fig. 30; Cymbephallus elongatus Yamaguti, 1940: 108; Pseudopecoelus elongatus: Wicklen, 1946: 159, 161; Pseudopecoelus elongatus: Manter \& Van Cleave, 1951: 322, 323; Pseudopecoelus elongatus: Yamaguti, 1954: 112; Pseudopecoelus elongatus: Manter, 1954: 507; Pseudopecoelus elongatus: Yamaguti, 1958: 170; Pseudopecoelus elongatus: Skrjabin \& Petrov, 1958: 296, 304, fig. 96; Pseudopecoelus elongatus: Yamaguti, 1964: 131; Pseudopecoelus elongatus: Travassos, Freitas e Buhrnheim, 1967: 16-18, est. 8, figs. 23-27; Pseudopecoelus elongatus: Travassos, Freitas e Kohn, 1969: 361, figs. 250-251.

Trematódeos de corpo alongado, extremidades arredondadas e cutícula não espinhosa; medem 1,76 a $2,51 \mathrm{~mm}$ de comprimento por 0,34 a $0,44 \mathrm{~mm}$ de largura. Ventosa oral subterminal com 0,12 a $0,16 \mathrm{~mm}$ de comprimento por 0,18 a $0,23 \mathrm{~mm}$ de largura. Acetábulo saliente, pedunculado ou embebido no corpo; mede 0,16 a $0,25 \mathrm{~mm}$ de comprimento por 0,23 a $0,27 \mathrm{~mm}$ de largura. Relação entre a ventosa oral e o acetábulo, varia de $1: 1,05$ a 1:1,31. Faringe musculosa, 


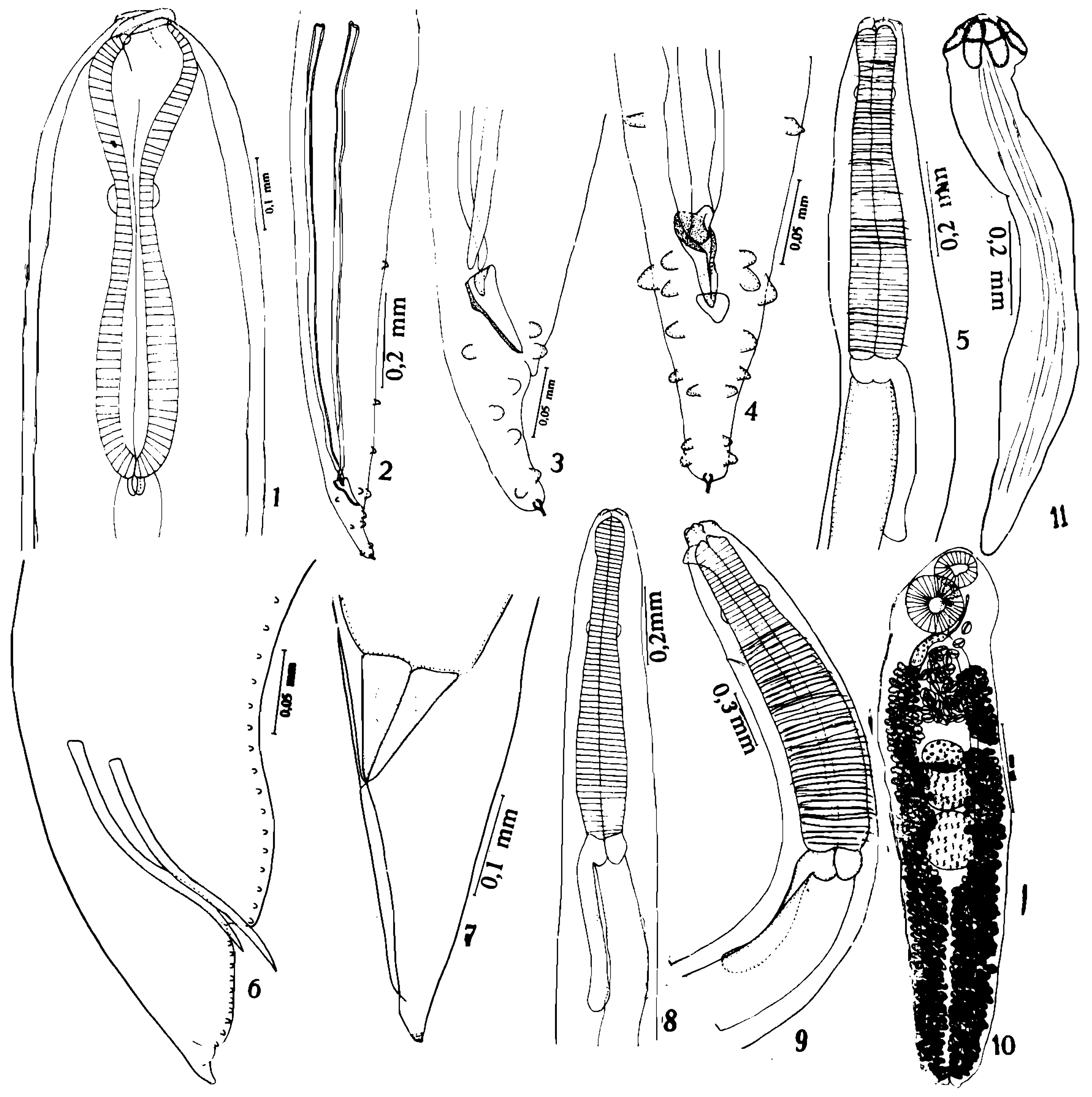

Cucullanus rougetae sp. n. - Fig. 1: extremidade anterior do holótipo n. 30 969; Fig. 2: extremidade posterior, vista lateral, do holótipo n. 030 969; Fig. 3: extremidade posterior, vista lateral do holótipo n. ${ }^{\circ} 30969$; Fig. 4: extremidade posterior, vista ventral do holótipo n. 030969.

Raphidascaris yamagutti sp. n. - Fig. 5: extremidade posterior do parátipo n.o 30970 a; Fig. 6: extremidade posterior, vista lateral, do holótipo macho n.o 30970 d; Fig. 7: extremidade posterior do holótipo fêmea n.o 30970 b.

Raphidascaris sp. 1 - Fig. 8: extremidade anterior do exemplar n.0 30971 a.

Raphidascaris sp. 2 - Fig. 9: extremidade anterior.

Pseudopecoelus elongatus (Yamaguti, 1938) Wicklen, 1946 - Fig. 10: exemplar n.o 30974 a. Original.

Phyllobothriidae sp. - Fig. 11: exemplar n.o 30975 c. 
com 0,11 a $0,13 \mathrm{~mm}$ de comprimento por 0,10 a $0,11 \mathrm{~mm}$ de largura. Esôfago curto. Cecos intestinais mais ou menos retilíneos, atingindo a extremidade posterior do corpo. Poro genital submediano na zona da faringe. Átrio genital curto. Bolsa do cirro ausente. Vesícula seminal presente, pós-ace tabular; mede 0,19 a $0,22 \mathrm{~mm}$ de comprimento por 0,070 a $0,080 \mathrm{~mm}$ de largura. Cirro curto inaparente. Testículos pós-acetabulares, pós-ovarianos, intercecais, situados no mesmo campo com zonas em contato ou um pouco afastadas. Testículo anterior com 0,15 a $0,23 \mathrm{~mm}$ de comprimento por 0,12 a $0,23 \mathrm{~mm}$ de largura; testículo posterior com 0,15 a $0,22 \mathrm{~mm}$ de comprimento por 0,15 a $0,25 \mathrm{~mm}$ de largura. Ovário intercecal, pós-acetabular e pré-testicular no campo dos testículos e com zona um pouco afastada ou parcialmente coincidente com a do testículo anterior; mede 0,11 a $0,16 \mathrm{~mm}$ de comprimento por 0,09 a $0,16 \mathrm{~mm}$ de largura. Glândula de Mehlis pré-ovariana. Espermateca ausente. Útero com poucos ovos, pré-ovariano e quase todo intercecal, ligando-se ao átrio genital por uma vagina não diferenciada. Ovos amarelos, operculados, com 0,050 a $0,060 \mathrm{~mm}$ de comprimento por 0,030 a $0,040 \mathrm{~mm}$ de largura. Vitelinos constituídos por folículos numerosos e arredondados, estendendo-se do limite posterior da zona da vesícula seminal até a extremidade posterior do corpo; são extracecais, cecais e intercecais interrompidos ao nível das gônadas ocupando a área intercecal pós-testicular. Poro excretor terminal. Vesícula excretora não observada.

Habitat - Intestino e cecos de Trichiurus lepturus $L$. Brasil.

Proveniência - Macaé, Estado do Rio de Janeiro,

Material estudado, depositado na Coleção Helmintológica do Instituto Oswaldo Cruz, sob o número 30974 a-j.

Referências bibliográficas $-9,10,17,18,21,24$, $26,27,28$ e 29.

Observação: Referido em novo hospedeiro.

\section{CESTODA}

Tetraphyllidea Carus, 1863

Phyllobothriidae sp.

(Fig. 11)

Exemplares de pequenas dimensões, afilados em sua porção terminal, médindo 2,40 a $3,08 \mathrm{~mm}$ de comprimento por 0,28 a $0,35 \mathrm{~mm}$ de largura. Escólex bem delimitado do resto do corpo, medindo 0,24 a $0,027 \mathrm{~mm}$ de comprimento por 0,34 a $0,39 \mathrm{~mm}$ de largura. Ventosas com 0,16 a $0,20 \mathrm{~mm}$ de comprimento por $0,10 \mathrm{~mm}$ de largura. Saco rostelar medindo $0,090 \mathrm{~mm}$ de comprimento por 0,072 a $0,090 \mathrm{~mm}$ de largura.

Habitat - Intestino de Urophycis sp. e Prionotus sp.
Proveniência - Macaé, Estado do Rio de Janeiro, Brasil.

A descrição acima é baseada em numerosos indivíduos jovens e, de um deles, foi feita a figura apresentada.

Material estudado, depositado na Coleção Helmintológica do Instituto Oswaldo Cruz sob os números 30975 a-c e 30 976 a-b.

Referência bibliográfica -30 .

\section{DISCUSSÃO}

1 - Dos 62 peixes examinados 11 se apresentaram parasitados por helmintos (aproximadamente $16,11 \%$ ). O parasitismo geral por grupo de helmintos é o seguinte:

Parasitados por nematódeos

5 exemplares ............

$50 \%$

Parasitados por trematódeos

3 exemplares

Parasitados por cestódeos (larvas)

2 exemplares ............

Observação - As percentagens dizem respeito apenas aos animais parasitados.

2 - Conforme observações anteriores, voltamos a constatar um índice baixo de parasitismo.

3 - São descritas 2 espécies de nematódeos: Cucullanus rougetae e Raphidascaris yamagutii

4 - As espécies Procamallanus macaensis Vicente \& Santos, 1972 e Pseudopecoelus elongatus (Yamaguti, 1938) Wicklen, 1946, são referidas em novos hospedeiros.

\section{SUMMARY}

Several helminths were recovered, by one of the authors (Elias dos Santos) from fishes obtained in Macaé Coast, Rio de Janeiro State, Brasil, during an excursion in January, 1973. Two new species are described: Cucullanus rougetae and Raphidascaris yamagutii; they 
redescribe and give new host records for Pseudopecoellus elongatus (Yamaguti, 1938) and Procamallanus macaensis Vicente e Santos, 1972; the later is only refered, as well as Catarinatrema verrucosum Freitas e Santos, 1971. They describe one cestode and two nematode larvae although they could not be determinated. This is the second publication on the matter.

\section{AGRADECIMENTOS}

Desejamos agradecer pela determinação dos peixes, aos técnicos do Museu Nacional e também aos pescadores da Cooperativa Mista dos Pescadores de Macaé, que gentilmente nos of ertaram os peixes para as necrópsias.

\section{REFERÊNCIAS BIBLIOGRÁFICAS}

1 - AGRAWAL, V., 1967, Some new Camallanoidea (Spirurida) nematodes from fishes, amphibians and reptiles. Ann. Parasit. Hum. Comp. 42 (3): $327-342,21$ figs.

2 - ALI, S. M., 1956, Studies on the nematode parasites of fishes and birds found in Hyderabad State. Indian Helminth, 8 (1): 1-83, 9 pls.

3 - CAMPANA-ROUGET, Y., 1957, Parasites de poissons de mer ouest-africains récoltés par $J$. Cadenat. Nematodes ( $4 \mathrm{e}$ note). Sur quelques espéces de Cucullanidae. Revision de la sousfamille. Bull. de v'I.F.A.N., 19 (2): 417470, 47 figs.

4 - CHANDLER, A., 1935, Parasites of fishes in Galveston bay. Proc. U.S. Nat. Mus., 83 (2977): 123-157, 56 figs.

5 - FREITAS, J. F. T. \& SANTOS, E., 1959, Novo trematódeo parasito de peixe marinho. Mem. Inst. Oswaldo Cruz, 69 (1): 79-81, 5 ests.

6 - LE-VAN-HOA \& PHAM-NGOC-KHUE, 1967, Morphologie et cycle évolutif de Cucullanus chabaudi n. sp., parasite des poissons Pangasius pangasius H. B. (P. Buchanani) du SudVietnam. Bull Soc. Path Exot., 60 (3): 315-318, 9 figs.

7 - LYSTER, L. L., 1940, Parasites of freswater fish II. Parasitism of speckled and lake trout and the fish found associated with them in lake commandant, Que. Canad. J. Res., 18 (2): 66-78, 20 figs.
8 - MAGGENTI, A. R., 1971, A review of the family Cucullanidae Cobbold, 1864 and the genus Bulbodacnitis Lane, 1916 with a description of Bulbodacnitis ampullostoma sp. n. (Nematoda: Cucullanidae) from Salmo gaird. nerii Richardson. Proc. Helm. Soc. Wash., 38 (1): 80-85, 6 figs.

9 - MANTER, H. W., 1954, Some digenctic trematodes from fishes of New Zealand. Trans. Roy. Soc. New Zealand, 82 (2): 475-568, 89 figs.

10 - MANTER, H.W. \& VAN CLEAVE, H. J., 1951, Some digenetic trematodes incluiding eight new species from marine fishes of $\mathrm{La}$ Jolla, California. Proc. U.S. Nat. Mus, 101 (3279): 315-340, pls. 12-13, 18 figs.

11 - MARGOLIS, L., 1960, A new nematode of the genus Cucullanus (Camallanata. Cucullanidae) from a flounder, Parophrys vetulus Girard, 1854 , with notes on the species from Pleuronectiformes. Canad J. Zool., 38 (5): 839-849, 13 figs.

12 - MOZGOVOI, A. A., 1953, Ascaridata dos animais $e$ do homem e doenças causadas por eles. Parte II. In Skrjabin, K. I., 1953, Princlpios de Nematodologia, 2, 616 pp, figs. 1-323, Akad. Nauk SSSR ed., Moscou (em russo).

13 - RASHEED, S., 1968, The nematodes of the genus Cucullanus Mueller, 1777, from the marine fish of Karachi coast. An Esc. Nac. Cienc. Biol Mexico, 15 (14): 23-59, 10 pls, 53 figs.

14 - RICHARDSON, L., 1937, Raphidascaris laurentianus sp. nov. (Ascaroidea) from Salvelinus fontinalis (Mitchill) in Quebec. Canad. J. Res., D., 15: 112-115, fig 1 .

15 - SANTOS, E., 1970, Nova espécie do gênero Raphidascaris Railliet \& Henry, 1915 (Nematoda, Ascaridoidea). Atas Soc Biol Rio de Janeiro, 14 (1-2): 39-40, 4 figs.

16 - SCHMIDT, G. D. \& KUNTZ, R. E., 1969, Nematode parasites of Oceanica. V. Four new species from fishes of Palawan, P.I. with a proposal for Oceanicucullanus gen. nov. Parasitology, 59 (2): 389-396, 11 figs.

17 - SKRJABIN, K. I., 1964, Keys to the trematodes of animals and man, XVI + 351 pp., 919 figs., Univ. III. Press end., Urbana.

18 - SKRJABIN, K. I. \& PETROV, A. M., 1958 , Subfamilia Opecoelinae Stunkard, 1931. In SKRJABIN, K. I., 1958, Trematodeos dos animais e do homem, Tratado de Trematodologia, 15, 820 pp, 277 figs, Akad. Nauk SSSR ed, Moscou (em russo). 
19 - SOOD, M. L., 1968, Some nematode parasites from fresh water fishes of India. Indian. $J$. Helminth., 20 (2): 83-110, 32 figs.

20 - TORNQUIST, N., 1931, Die Nematodenfamilien Cucullanidae und Camallanidae, nobst weitere Beitraegen zur Kenntnis der Anatomie und Histologie der Nematoden. Goteborgs Vetensk-Samh. Hanse (5 B), 2: 1441, 17 taf., figs.

21 - TRAVASSOS, L., FREITAS, J. F. T. \& BUHRNHEIM, P. F., 1967, Relatório da Excursão do Instituto Oswaldo Cruz ao Estado do Espírito Santo em novembro de 1964. Bol. Mus. Biol Mello Leitão, Zoologia, (31): 1-54, 21 ests, 75 figs.

22 - VICENTE, J. J. \& SANTOS, E., 1972, Sobre um novo nematódeo camalanídeo parasito de peixe marinho (Nematoda, Camallanoidea). Atas Soc. Biol. Rio de Janeiro, 15 (3): 145-147, 5 figs.

23 - VICENTE, J. J. \& SANTOS, E., 1973, Alguns helmintos de peixes do litoral norte fluminense - I. Mem Inst. Oswaldo Cruz, 71 (1-2): 95-113, 12 figs.

24 - WICKLEN, J.H., 1946, The trematode genus Opecoeloides and related genera with a descrip- tion of Opecoeloides polynemi n. sp. J. Parasitol, 32 (2): 156-163, 7 figs.

25 - YAMAGUTl, S., 1935, Studies on the helminth fauna of Japan. Part 9. Nematodes of fishes I. Jap. J. Zool, 6: 337-386, 65 figs.

26 - YAMAGUTI, S., 1938, Studies on the helminth fauna of Japan Part 21. Trematodes of fishes, IV, 139 pp., 83 figs., 1 pl., 9 figs., author ed. Kyoto.

27 - YAMAGUTI, S., 1940, Studies on the helminth fauna of Japan. Part 31. Trematodes of fishes, VII. Jap. J. Zool., 9 (1): 36-108, 51 figs., 2 pls., 12 figs.

28 - YAMAGUTI, S., 1954, Systema Helminthum, Part I, Digenetic trematodes of fishes, (1953), II +405 pp, 11 figs, 32 pls., 422 figs, author ed., Tokyo.

29 - YAMAGUTI, S., 1958, Systema Helminthum, 1, The digenetic trematodes of vertebrates, Part I: XI + 979 pp., Part II: 980-1232, 1445-1575, 106 pls, 1302 figs, Interscience Publishers, Inc. ed, New York.

30 - YAMAGUTI, S, 1959, Systema Helminthum, 2, The Cestodes of vertebrates: 1-626, 769-860, 70 pls, 584 figs. Interscience Publishers, Inc. ed., New York. 